\title{
Regularity and Green's Relations for Generalized Semigroups of Transformations with Invariant Set
}

\author{
Lei $\operatorname{Sun}^{1}$ \\ ${ }^{1}$ School of Mathematics and Information Science, Henan Polytechnic University, P.R.China \\ Correspondence: Lei Sun, School of Mathematics and Information Science, Henan Polytechnic University, Henan, \\ Jiaozuo, 454003, P.R.China. E-mail: sunlei97@163.com
}

Received: June 30, 2017 Accepted: August 16, 2017 Online Published: February 6, 2018

doi:10.5539/jmr.v10n2p24 URL: https://doi.org/10.5539/jmr.v10n2p24

\begin{abstract}
Let $\mathcal{T}_{X}$ be the full transformation semigroup on a set $X$. For $Y \subseteq X$, the semigroup $S(X, Y)=\left\{f \in \mathcal{T}_{X}: f(Y) \subseteq Y\right\}$ is a subsemigroup of $\mathcal{T}_{X}$. Fix an element $\theta \in S(X, Y)$ and for $f, g \in S(X, Y)$, define a new operation $*$ on $S(X, Y)$ by $f * g=f \theta g$ where $f \theta g$ denotes the produce of $g, \theta$ and $f$ in the original sense. Under this operation, the semigroup $S(X, Y)$ forms a semigroup which is called generalized semigroup of $S(X, Y)$ with the sandwich function $\theta$ and denoted by $S\left(X, Y, *_{\theta}\right)$. In this paper we first characterize the regular elements and then describe Green's relations for the $\operatorname{semigroup} S\left(X, Y, *_{\theta}\right)$.
\end{abstract}

Keywords: generalized transformation semigroups, regular elements, Green's relations

2010 Mathematics Subject Classification: 20M20

\section{Introduction}

Let $S$ be a semigroup and $a, b \in S$. If $a=a x a$ for some $x \in S$, then $a$ is called a regular element of $S$. The semigroup $S$ is called regular if all its elements are regular. If $a$ and $b$ generate the same left principle ideal, that is, $S^{1} a=S^{1} b$, then we say that $a$ and $b$ are $\mathcal{L}$ equivalent and write $(a, b) \in \mathcal{L}$ or $a \mathcal{L} b$. If $a$ and $b$ generate the same right principle ideal, that is, $a S^{1}=b S^{1}$, then we say that $a$ and $b$ are $\mathcal{R}$ equivalent and write $(a, b) \in \mathcal{R}$ or $a \mathcal{R} b$. If $a$ and $b$ generate the same principle ideal, that is, $S^{1} a S^{1}=S^{1} b S^{1}$, then we say that $a$ and $b$ are $\mathcal{J}$ equivalent and write $(a, b) \in \mathcal{J}$ or $a \mathcal{J} b$. It is not difficult to see that $\mathcal{L}, \mathcal{R}$ and $\mathcal{J}$ are equivalence relations on $S$. Let $\mathcal{H}=\mathcal{L} \cap \mathcal{R}$ and $\mathcal{D}=\mathcal{L} \vee \mathcal{R}$. Then $\mathcal{H}$ and $\mathcal{D}$ are also equivalences. These five equivalences are usually called Green's relations on $S$. They were introduced by J.A. Green and play an important role in the study of the algebraic structure of semigroups.

Let $\mathcal{T}_{X}$ be the full transformation semigroup on a set $X$. Given a subset $Y$ of $X$, the authors in (Honyam, P. \& Sanwong, J., 2011) observed a class of subsemigroup of $\mathcal{T}_{X}$ defined by

$$
S(X, Y)=\left\{f \in \mathcal{T}_{X}: f(Y) \subseteq Y\right\}
$$

It is clear that if $Y=X$ then $S(X, Y)=\mathcal{T}_{X}$. To this extent the semigroup $S(X, Y)$ is regarded as a generalization of $\mathcal{T}_{X}$. Regularity for the elements in $S(X, Y)$ and Green's relations on $S(X, Y)$ were described in (Honyam, P. \& Sanwong, J., 2011).

We apply transformations on the left so that for $f, g \in S(X, Y)$, their product $f g$ is the transformation obtained by first performing $g$ and then $f$. Fix an element $\theta \in S(X, Y)$ and for $f, g \in S(X, Y)$, define a new operation $*$ on $S(X, Y)$ by $f * g=f \theta g$ where $f \theta g$ denotes the produce of $g, \theta$ and $f$ in the original sense. Under this operation, the semigroup $S(X, Y)$ forms a semigroup which is called generalized semigroup of $S(X, Y)$ with the sandwich function $\theta$ and denoted by $S\left(X, Y, *_{\theta}\right)$. Then $S\left(X, Y, *_{\theta}\right)=S(X, Y)$ as sets. Moreover, if $\theta=\mathrm{id}_{X}$ (the identity transformation on the set $\left.X\right)$, then $S\left(X, Y, *_{\theta}\right)=S(X, Y)$ as semigroups. The generalized transformation semigroups of the various subsemigroups of $\mathcal{T}_{X}$ were studied by many authors, see for example (Hickey, J. B., 1983; Kemprasit, Y. \& Jaidee, S., 2005; Magill, K. D. Jr. \& Subbiah, S., 1975; Pei, H. S., Sun, L. \& Zhai, H. C., 2007; Symons, J. S., 1975; Tsyaputa, G. Y., 2004).

The purpose of this paper is to investigate the regularity of elements and Green's relations on generalized semigroup $S\left(X, Y, *_{\theta}\right)$. Accordingly, in Section 2 , the condition under which an element $f \in S\left(X, Y, *_{\theta}\right)$ is regular is analyzed. In Section 3, Green's relations on $S\left(X, Y, *_{\theta}\right)$ are considered and the relations $\mathcal{L}, \mathcal{R}, \mathcal{H}, \mathcal{D}$ and $\mathcal{J}$ are descried for arbitrary elements, respectively. 


\section{The Regular Elements of $S\left(X, Y, *_{\theta}\right)$}

In this section we investigate the condition under which an element of $S\left(X, Y, *_{\theta}\right)$ is regular.

Theorem 2.1. Let $f \in S\left(X, Y, *_{\theta}\right)$. Then $f$ is regular if and only if the following statements hold.

(1) $\left.\theta\right|_{f(X)}$ is injective.

(2) $\theta f(X)=\theta f \theta(X)$ and $\theta f(X) \cap Y=\theta f \theta(Y)$.

Proof. Suppose that $f$ is regular. Then $f=f * g * f=f \theta g \theta f$ for some $g \in S\left(X, Y, *_{\theta}\right)$. It follows that $\left.(f \theta)(g \theta)\right|_{f(X)}=\left.\mathrm{id}\right|_{f(X)}$ and $\left.(g \theta)\right|_{f(X)}$ is injective. So $\left.\theta\right|_{f(X)}$ is injective and (1) holds. Clearly, $\theta f \theta(X) \subseteq \theta f(X)$. For each $z \in \theta f(X)$, let $z=\theta f(x)$ for some $x \in X$. Write $y=g \theta f(x)$ and then $z=\theta f(x)=\theta f \theta g \theta f(x)=\theta f \theta(y)$ which implies that $\theta f(X) \subseteq \theta f \theta(X)$. Thus $\theta f(X)=\theta f \theta(X)$. Similarly, we have $\theta f(X) \cap Y=\theta f \theta(Y)$.

Conversely, assume that (1)-(2) hold. Then, for each $x \in \theta f(X) \cap Y$, let $x=\theta f \theta(y)$ for some $y \in Y$, and for each $x \in \theta f(X)-Y$, let $x=\theta f \theta\left(y^{\prime}\right)$ for some $y^{\prime} \in X$. Arbitrarily fix $a \in Y$ and define $g: X \rightarrow X$ by

$$
g(x)= \begin{cases}y & \text { if } x \in \theta f(X) \cap Y \\ y^{\prime} & \text { if } x \in \theta f(X)-Y \\ a & \text { otherwise. }\end{cases}
$$

Clearly, $g \in S\left(X, Y, *_{\theta}\right)$. To see $f=f \theta g \theta f$, we need only to show that $\theta f=\theta f \theta g \theta f$ since $\left.\theta\right|_{f(X)}$ is injective. For each $x \in X$, if $\theta f(x) \in \theta f(X) \cap Y$, then let $\theta f(x)=\theta f \theta(y)$ for some $y \in Y$. If $\theta f(x) \in \theta f(X)-Y$, then let $\theta f(x)=\theta f \theta\left(y^{\prime}\right)$ for some $y^{\prime} \in X$. So

$$
\begin{aligned}
& \theta f \theta g \theta f(x)= \begin{cases}\theta f \theta(y) & \text { if } \theta f(x) \in \theta f(X) \cap Y \\
\theta f \theta\left(y^{\prime}\right) & \text { if } \theta f(x) \in \theta f(X)-Y\end{cases} \\
& =\theta f(x)
\end{aligned}
$$

which means that $\theta f=\theta f \theta g \theta f$ and so $f=f \theta g \theta f$. Therefore $f$ is regular.

Denote by $\operatorname{Reg}\left(S\left(X, Y, *_{\theta}\right)\right)$ and $\operatorname{Reg}(S(X, Y))$ the sets of all regular elements in semigroups $S\left(X, Y, *_{\theta}\right)$ and $S(X, Y)$, respectively. It is clear that $\operatorname{Reg}\left(S\left(X, Y, *_{\theta}\right)\right) \subseteq \operatorname{Reg}(S(X, Y))$. In generally, an element $f \in \operatorname{Reg}(S(X, Y))$ may be not regular in $S\left(X, Y, *_{\theta}\right)$. The following theorem shows when $\operatorname{Reg}\left(S\left(X, Y, *_{\theta}\right)\right)=\operatorname{Reg}(S(X, Y))$.

Theorem 2.2. Let the sets $\operatorname{Reg}\left(S\left(X, Y, *_{\theta}\right)\right)$ and $\operatorname{Reg}(S(X, Y))$ be defined as above. Then $\operatorname{Reg}\left(S\left(X, Y, *_{\theta}\right)\right)=\operatorname{Reg}(S(X, Y))$ if and only if $\theta$ is a bijection and $\theta(X-Y) \cap Y=\emptyset$.

Proof. Suppose that $\operatorname{Reg}\left(S\left(X, Y, *_{\theta}\right)\right)=\operatorname{Reg}(S(X, Y))$. Since the identity transformation $\operatorname{id}_{X}$ on $X$ is regular in $S(X, Y)$, we have that $\mathrm{id}_{X}$ is also regular in $S\left(X, Y, *_{\theta}\right)$, that is, $\mathrm{id}_{X}=\operatorname{id}_{X} \theta g \theta \mathrm{id}_{X}=\theta g \theta$ for some $g \in S\left(X, Y, *_{\theta}\right)$. So $\theta$ is bijective. Now we assert that $\theta(X-Y) \cap Y=\emptyset$. Indeed, if $\theta(x) \in Y$ for some $x \in X-Y$, then $x=\operatorname{id}_{X}(x)=\theta g \theta(x) \in Y$, a contradiction. Therefore, $\theta(X-Y) \cap Y=\emptyset$.

Conversely, we need to show that $\operatorname{Reg}(S(X, Y)) \subseteq \operatorname{Reg}\left(S\left(X, Y, *_{\theta}\right)\right)$. For this purpose, let $f \in \operatorname{Reg}(S(X, Y))$. Then $f=f g f$ for some $g \in S(X, Y)$. Since $\theta$ is a bijection and $\theta(X-Y) \cap Y=\emptyset$, it follows that $\theta^{-1}(Y) \subseteq Y$. So $\theta^{-1} \in S\left(X, Y, *_{\theta}\right)$ and $g^{\prime}=$ $\theta^{-1} g \theta^{-1} \in S\left(X, Y, *_{\theta}\right)$. Thus $f=f \theta g^{\prime} \theta f$ which implies that $f \in \operatorname{Reg}\left(S\left(X, Y, *_{\theta}\right)\right)$. Hence $\operatorname{Reg}(S(X, Y)) \subseteq \operatorname{Reg}\left(S\left(X, Y, *_{\theta}\right)\right)$ and $\operatorname{Reg}(S(X, Y))=\operatorname{Reg}\left(S\left(X, Y, *_{\theta}\right)\right)$.

Theorem 2.3. The semigroup $S\left(X, Y, *_{\theta}\right)$ is regular if and only if the following statements hold.

(1) $\theta$ is a bijection and $\theta(X-Y) \cap Y=\emptyset$.

(2) $Y=X$ or $|Y|=1$.

Proof. Suppose that $S\left(X, Y, *_{\theta}\right)$ is regular. Then

$$
S\left(X, Y, *_{\theta}\right)=\operatorname{Reg}\left(S\left(X, Y, *_{\theta}\right)\right) \subseteq \operatorname{Reg}(S(X, Y)) \subseteq S(X, Y) .
$$

Since $S\left(X, Y, *_{\theta}\right)=S(X, Y)$ as sets, it follows that $\operatorname{Reg}\left(S\left(X, Y, *_{\theta}\right)\right)=\operatorname{Reg}(S(X, Y))$. By Theorem 2.2, $\theta$ is a bijection and $\theta(X-Y) \cap Y=\emptyset$. In the meantime, the semigroup $S(X, Y)$ is also regular. By [5, Corollary 2.4], $Y=X$ or $|Y|=1$.

Conversely, by [5, Corollary 2.4] and Theorem 2.2, we have

$$
S(X, Y)=\operatorname{Reg}(S(X, Y))=\operatorname{Reg}\left(S\left(X, Y, *_{\theta}\right)\right) \subseteq S\left(X, Y, *_{\theta}\right) .
$$

Since $S\left(X, Y, *_{\theta}\right)=S(X, Y)$ as sets, it follows that $S\left(X, Y, *_{\theta}\right)=\operatorname{Reg}\left(S\left(X, Y, *_{\theta}\right)\right)$, as required. 


\section{Green's Relations on $S\left(X, Y, *_{\theta}\right)$}

In this section we describe Green's relations on $S\left(X, Y, *_{\theta}\right)$.

Denote by $\pi(f)$ the partition of $X$ induced by $f \in \mathcal{T}_{X}$, namely,

$$
\pi(f)=\left\{f^{-1}(y): y \in f(X)\right\} .
$$

Also, let

$$
\pi_{Y}(f)=\{P \in \pi(f): P \cap Y \neq \emptyset\} .
$$

Let $\psi: \pi(f) \rightarrow \pi(g)$ be a map. If $\theta(X) \cap \psi(P) \neq \emptyset$ for each $P \in \pi(f)-\pi_{Y}(f)$ and $\theta(Y) \cap \psi(P) \neq \emptyset$ for each $P \in \pi_{Y}(f)$, then $\psi$ is said to be $\theta_{Y}$-admissible. If $\psi$ is bijective and both $\psi$ and $\psi^{-1}$ are $\theta_{Y}$-admissible, then $\psi$ is said to be $\theta_{Y}^{*}$-admissible.

Now we begin with the relation $\mathcal{L}$ in $S\left(X, Y, *_{\theta}\right)$.

Theorem 3.1. Let $f, g \in S\left(X, Y, *_{\theta}\right)$. Then the following statements are equivalent.

(1) $(f, g) \in \mathcal{L}$.

(2) $f(X)=g \theta(X), f(Y)=g \theta(Y)$ and $g(X)=f \theta(X), g(Y)=f \theta(Y)$.

(3) There is a $\theta_{Y}^{*}$-admissible bijection $\psi: \pi(f) \rightarrow \pi(g)$ such that $f=g \psi$.

Proof. $(1) \Longrightarrow(2)$. Suppose that $(f, g) \in \mathcal{L}$. Then $f=g \theta h$ and $g=f \theta k$ for some $h, k \in S\left(X, Y, *_{\theta}\right)$ and

$$
f(X)=g \theta h(X) \subseteq g \theta(X)=f \theta k \theta(X) \subseteq f(X),
$$

which implies that $f(X)=g \theta(X)$. Moreover,

$$
f(Y)=g \theta h(Y) \subseteq g \theta(Y)=f \theta k \theta(Y) \subseteq f(Y),
$$

which implies that $f(Y)=g \theta(Y)$. Similarly, $g(X)=f \theta(X)$ and $g(Y)=f \theta(Y)$.

(2) $\Longrightarrow(3)$. It is readily consequential on (2) that $f(X)=g(X)$. Now define $\psi: \pi(f) \rightarrow \pi(g)$ as follows. For each $P \in \pi(f)$, let $\psi(P)=g^{-1}(f(P))$. Then $\psi$ is a well-defined bijection and $f=g \psi$. To see that $\psi: \pi(f) \rightarrow \pi(g)$ is $\theta_{Y}^{*}$-admissible, let $\pi(f)=\left\{P_{i}: i \in I\right\}$ (where $I$ is some index set) and $x_{i}=f\left(P_{i}\right)(i \in I)$. If $P_{i} \cap Y=\emptyset$, then $x_{i} \in f(X)=g \theta(X)$ and $x_{i}=g\left(y_{i}\right)$ for some $y_{i} \in \theta(X)$. So $y_{i} \in \theta(X) \cap g^{-1}\left(x_{i}\right)$ and

$$
\theta(X) \cap \psi\left(P_{i}\right)=\theta(X) \cap g^{-1}\left(f\left(P_{i}\right)\right)=\theta(X) \cap g^{-1}\left(x_{i}\right) \neq \emptyset .
$$

If $P_{i} \cap Y \neq \emptyset$, then $x_{i} \in f(Y)=g \theta(Y)$ and $x_{i}=g\left(y_{i}\right)$ for some $y_{i} \in \theta(Y)$. Thus $y_{i} \in \theta(Y) \cap g^{-1}\left(x_{i}\right)$ and

$$
\theta(Y) \cap \psi\left(P_{i}\right)=\theta(Y) \cap g^{-1}\left(f\left(P_{i}\right)\right)=\theta(Y) \cap g^{-1}\left(x_{i}\right) \neq \emptyset .
$$

Hence $\psi: \pi(f) \rightarrow \pi(g)$ is $\theta_{Y}$-admissible. Similarly, $\psi^{-1}$ is also $\theta_{Y}$-admissible. Consequently, $\psi: \pi(f) \rightarrow \pi(g)$ is a $\theta_{Y}^{*}$-admissible bijection.

(3) $\Longrightarrow(1)$. Suppose that (3) holds. For each $x \in X$, if $P_{x}=f^{-1}(f(x)) \cap Y \neq \emptyset$, then take $z \in \theta(Y) \cap \psi\left(P_{x}\right)$ and let $z=\theta(y)$ for some $y \in Y$. Define $h(x)=y$. If $P_{x}=f^{-1}(f(x)) \cap Y=\emptyset$, then take $z \in \theta(X) \cap \psi\left(P_{x}\right)$ and let $z=\theta(y)$ for some $y \in X$. Define $h(x)=y$. Clearly, $h \in S\left(X, Y, *_{\theta}\right)$. To see that $f=g \theta h$, for each $x \in X$, let $P_{x}=f^{-1}(f(x))$ and $Q_{z}=g^{-1}(g(z))$ (where $z \in \theta(Y) \cap \psi\left(P_{x}\right)$ or $z \in \theta(X) \cap \psi\left(P_{x}\right)$ ), then

$$
f(x)=f\left(P_{x}\right)=g \psi\left(P_{x}\right)=g\left(Q_{z}\right)=g\left(Q_{\theta(y)}\right)=g\left(Q_{\theta(h(x))}\right)=g \theta h(x)
$$

and so $f=g \theta h$. Similarly, $g=f \theta k$ for some $k \in S\left(X, Y, *_{\theta}\right)$. Therefore, $(f, g) \in \mathcal{L}$.

Let $Z$ be a subset of $X$ and $Z \cap Y \neq \emptyset$. Let $\phi: Z \rightarrow X$ be a map. If $\phi(Z \cap Y) \subseteq Y$, then $\phi$ is said to be $Y$-variant. Clearly, each transformation $f \in S\left(X, Y, *_{\theta}\right)$ is Y-variant. If $\phi$ is bijective and both $\phi$ and $\phi^{-1}$ are Y-variant, then $\phi$ is said to be $Y^{*}$-variant.

Now we consider the relation $\mathcal{R}$.

Theorem 3.2. Let $f, g \in S\left(X, Y, *_{\theta}\right)$. Then the following statements are equivalent.

(1) $(f, g) \in \mathcal{R}$.

(2) $\pi(\theta f)=\pi(f)=\pi(g)=\pi(\theta g)$ and $\pi_{Y}(\theta f)=\pi_{Y}(f)=\pi_{Y}(g)=\pi_{Y}(\theta g)$. 
(3) There is a $Y^{*}$-variant bijection $\phi: f(X) \rightarrow g(X)$ such that $g=\phi f$, and $\left.\theta\right|_{f(X)}$ and $\left.\theta\right|_{g(X)}$ are injective. Moreover, $\theta f(x) \in Y \Rightarrow f(x) \in Y$ and $\theta g\left(x^{\prime}\right) \in Y \Rightarrow g\left(x^{\prime}\right) \in Y$ for some $x, x^{\prime} \in X$.

Proof. $(1) \Longrightarrow(2)$. Suppose that $(f, g) \in \mathcal{R}$. Then $f=h \theta g$ and $g=k \theta f$ for some $h, k \in S\left(X, Y, *_{\theta}\right)$. Immediately, $\pi(f)=\pi(g)$ and $\pi_{Y}(f)=\pi_{Y}(g)$. By $f=h \theta k \theta f,\left.(h \theta)(k \theta)\right|_{f(X)}=\left.\mathrm{id}\right|_{f(X)}$ and $\left.(k \theta)\right|_{f(X)}$ is injective. It follows that $\left.\theta\right|_{f(X)}$ is injective and $\pi(\theta f)=\pi(f)$. Similarly, $\pi(\theta g)=\pi(g)$. Thus $\pi(\theta f)=\pi(f)=\pi(g)=\pi(\theta g)$. Now we verify that $\pi_{Y}(\theta f)=\pi_{Y}(f)$. Clearly, $\pi_{Y}(f)$ refines $\pi_{Y}(\theta f)$. Let $\theta f(x)=\theta f(y) \in Y$ for some distinct $x, y \in X$. Then, by $\pi(\theta f)=\pi(f)$ and $f=h \theta k \theta f, f(x)=f(y)=h \theta k \theta f(y) \in Y$. So $\pi_{Y}(\theta f)$ refines $\pi_{Y}(f)$ and $\pi_{Y}(\theta f)=\pi_{Y}(f)$. Also, we have $\pi_{Y}(\theta g)=\pi_{Y}(g)$. Consequently, $\pi_{Y}(\theta f)=\pi_{Y}(f)=\pi_{Y}(g)=\pi_{Y}(\theta g)$.

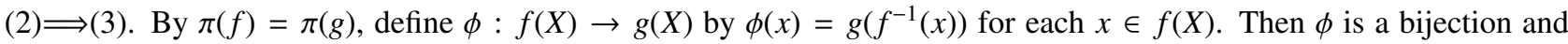
$g=\phi f$. Arbitrarily take $y \in f(X) \cap Y$. Then $f^{-1}(y) \in \pi_{Y}(f)=\pi_{Y}(g)$ and $\phi(y)=g\left(f^{-1}(y)\right) \in Y$ which implies that $\phi$ is Y-variant. Similarly, $\phi^{-1}$ is also Y-variant. Thus $\phi$ is $Y^{*}$-variant. In virtue of $\pi(\theta f)=\pi(f),\left.\theta\right|_{f(X)}$ is injective. Now assume that $\theta f(x) \in Y$ for some $x \in X$. Then there is some $P \in \pi_{Y}(\theta f)$ such that $x \in P$. It follows that from $\pi_{Y}(\theta f)=\pi_{Y}(f)$ that $f(x)=f(P) \in Y$. The argument for $g$ is the same.

(3) $\Longrightarrow(1)$. Suppose that (3) holds. For each $x \in \theta f(X) \cap Y$, let $x=\theta f\left(x^{\prime}\right)$ for some $x^{\prime} \in X$. Fix $a \in Y$ and define $k: X \rightarrow X$ by

$$
k(x)= \begin{cases}\phi\left(f\left(x^{\prime}\right)\right) & \text { if } x \in \theta f(X) \cap Y \\ a & \text { otherwise }\end{cases}
$$

If $x=\theta f\left(x^{\prime \prime}\right)$ for some $x^{\prime \prime} \in X$ and $x^{\prime \prime} \neq x^{\prime}$, then $f\left(x^{\prime}\right)=f\left(x^{\prime \prime}\right)$ since $\left.\theta\right|_{f(X)}$ is injective and $\phi\left(f\left(x^{\prime}\right)\right)=\phi\left(f\left(x^{\prime \prime}\right)\right)$. Thus $k$ is well-defined. We now show that $k \in S\left(X, Y, *_{\theta}\right)$. For each $y \in Y$, either $y \notin \theta f(X)$ or $y \in \theta f(X)$. If $y \notin \theta f(X)$, then $k(y)=a \in Y$. If $y \in \theta f(X)$, let $y=\theta f(x) \in Y$ for some $x \in X$ and then $f(x) \in Y$. So $k(y)=\phi(f(x)) \in Y$ since the map $\phi$ is Y-variant. Thus $k \in S\left(X, Y, *_{\theta}\right)$. One can show $g=k \theta f$. Similarly, $f=h \theta g$ for some $h \in S\left(X, Y, *_{\theta}\right)$. Therefore, $(f, g) \in \mathcal{R}$.

According to Theorems 3.1 and 3.2, we have the following conclusion readily.

Theorem 3.3. Let $f, g \in S\left(X, Y, *_{\theta}\right)$. Then the following statements are equivalent.

(1) $(f, g) \in \mathcal{H}$.

(2) $f(X)=g \theta(X), f(Y)=g \theta(Y), g(X)=f \theta(X), g(Y)=f \theta(Y)$, and $\pi(\theta f)=\pi(f)=\pi(g)=\pi(\theta g), \pi_{Y}(\theta f)=\pi_{Y}(f)=$ $\pi_{Y}(g)=\pi_{Y}(\theta g)$.

(3) There is a $\theta^{*}$-admissible bijection $\psi: \pi(f) \rightarrow \pi(g)$ such that $f=g \psi$, and while there is a $Y^{*}$-variant bijection $\phi: f(X) \rightarrow g(X)$ such that $g=\phi f$, and $\left.\theta\right|_{f(X)}$ and $\left.\theta\right|_{g(X)}$ are injective. Moreover, $\theta f(x) \in Y \Rightarrow f(x) \in Y$ and $\theta g\left(x^{\prime}\right) \in Y \Rightarrow$ $g\left(x^{\prime}\right) \in Y$ for some $x, x^{\prime} \in X$.

In what follows we describe the relation $\mathcal{D}$.

Theorem 3.4. Let $f, g \in S\left(X, Y, *_{\theta}\right)$. Then the following statements are equivalent.

(1) $(f, g) \in \mathcal{D}$.

(2) There are a $\theta^{*}$-admissible bijection $\psi: \pi(g) \rightarrow \pi(f)$ and a $Y^{*}$-variant bijection $\phi: g(X) \rightarrow f(X)$ such that $f \psi=\phi g$, $\left.\theta\right|_{f(X)}$ and $\left.\theta\right|_{g(X)}$ are injective. Moreover, $\theta f(x) \in Y \Rightarrow f(x) \in Y$ and $\theta g\left(x^{\prime}\right) \in Y \Rightarrow g\left(x^{\prime}\right) \in Y$ for some $x, x^{\prime} \in X$.

Proof. $(1) \Longrightarrow(2)$. Suppose that $(f, g) \in \mathcal{D}$. Then $(f, h) \in \mathcal{L}$ and $(h, g) \in \mathcal{R}$ for some $h \in S\left(X, Y, *_{\theta}\right)$. By $(f, h) \in \mathcal{L}$, $h(X)=f(X)$ and there is a $\theta^{*}$-admissible bijection $\psi: \pi(h) \rightarrow \pi(f)$ such that $h=f \psi$. By $(h, g) \in \mathcal{R}, \pi(h)=\pi(g)$ and there is a $Y^{*}$-variant bijection $\phi: g(X) \rightarrow h(X)$ such that $h=\phi g,\left.\theta\right|_{h(X)}$ and $\left.\theta\right|_{g(X)}$ are injective, and $\theta h(x) \in Y \Rightarrow h(x) \in Y$ and $\theta g\left(x^{\prime}\right) \in Y \Rightarrow g\left(x^{\prime}\right) \in Y$. Replacing $\pi(h)$ by $\pi(g)$ and $h(X)$ by $f(X)$, the domain of $\psi$ and the image of $\phi$ become respectively the required ones and $\left.\theta\right|_{f(X)}$ is injective as well. Now let $\theta f(x) \in Y$, then $\theta f(x)=\theta h\left(x^{\prime}\right)$ for some $x^{\prime} \in X$ and so $f(x)=h\left(x^{\prime}\right) \in Y$. From $\pi(h)=\pi(g)$ and $h=\phi g$, it follows that $h=\phi g$. Therefore, $f \psi=h=\phi g$.

(2) $\Longrightarrow(1)$. Define $h(x)=\phi(g(x))$ for each $x \in X$. Clearly, $h \in S\left(X, Y, *_{\theta}\right)$ and $h=\phi g$. Then $\pi(h)=\pi(g)$ and $h=\phi g=f \psi$. By Theorem 3.1, $(h, f) \in \mathcal{L}$ and $h(X)=f(X)$. So $\phi: g(X) \rightarrow h(X)$ is a $Y^{*}$-variant bijection such that $h=\phi g,\left.\theta\right|_{h(X)}$ is injective, and $\theta h(x) \in Y \Rightarrow h(x) \in Y$. By Theorem 3.2, $(h, g) \in \mathcal{R}$. Consequently, $(f, g) \in \mathcal{D}$.

Finally, we investigate the relation $\mathcal{J}$.

Lemma 3.5. Let $f, g \in S\left(X, Y *_{\theta}\right)$. Then $f=h \theta g \theta k$ for some $h, k \in S\left(X, Y, *_{\theta}\right)$ if and only if there is a Y-variant map $\phi: \theta g \theta(X) \rightarrow f(X)$ such that $f(X)=\phi(\theta g \theta(X))$ and $f(Y)=\phi(\theta g \theta(Y))$. 
Proof. Suppose that $f=h \theta g \theta k$. Arbitrarily fix $a \in h \theta g \theta k(Y)$ and then define $\phi: \theta g \theta(X) \rightarrow f(X)$ by

$$
\phi(x)= \begin{cases}h(x) & \text { if } x \in \theta g \theta k(X) \\ a & \text { if } x \in \theta g \theta(X)-\theta g \theta k(X) .\end{cases}
$$

It is clear that $\phi(\theta g \theta(X)) \subseteq f(X)$. Now take $y \in f(X)$ such that $y=f(x)$ for some $x \in X$. Write $k(x)=x^{\prime} \in X$. Then

$$
y=f(x)=h \theta g \theta k(x)=\phi(\theta g \theta k(x))=\phi\left(\theta g \theta\left(x^{\prime}\right)\right) .
$$

So $f(X) \subseteq \phi(\theta g \theta(X))$ and $f(X)=\phi(\theta g \theta(X))$. Similarly, we have $f(Y)=\phi(\theta g \theta(Y))$. In what follows we show that $\phi$ is Y-variant. Let $y \in \theta g \theta(X) \cap Y$. If $y \in \theta g \theta k(X) \cap Y$, then $\phi(y)=h(y) \in Y$. If $y \in(\theta g \theta(X)-\theta g \theta k(X)) \cap Y$, then $\phi(x)=a \in h \theta g \theta k(Y) \subseteq Y$. Therefore, $\phi$ is Y-variant.

Conversely, suppose that (1)-(2) hold. Arbitrarily fix $a \in Y$ and define $h: X \rightarrow X$ as follows.

$$
h(x)= \begin{cases}\phi(x) & \text { if } x \in \theta g \theta(X) \\ a & \text { otherwise. }\end{cases}
$$

It is clear that $h \in S\left(X, Y, *_{\theta}\right)$. By the hypothesis, for each $x \in Y$, there is some $y \in Y$ such that $f(x)=\phi(\theta g \theta(y))$ and each $x \in X-Y$, there is some $z \in X$ such that $f(x)=\phi(\theta g \theta(z))$. Define

$$
k(x)= \begin{cases}y & \text { if } x \in Y \\ z & \text { if } x \in X-Y .\end{cases}
$$

It is routine to show that $k \in S\left(X, Y, *_{\theta}\right)$ and $f=h \theta g \theta k$.

Theorem 3.6. Let $f, g \in S\left(X, Y, *_{\theta}\right)$. Then $(f, g) \in \mathcal{T}$ if and only if there are Y-variant maps $\phi: \theta g \theta(X) \rightarrow f(X)$ and $\psi: \theta f \theta(X) \rightarrow g(X)$ such that $f(X)=\phi(\theta g \theta(X)), f(Y)=\phi(\theta g \theta(Y))$ and $g(X)=\psi(\theta f \theta(X)), g(Y)=\psi(\theta f \theta(Y))$.

\section{Acknowledgements}

We would like to thank the referee for his/her valuable suggestions and comments which help to improve the presentation of this paper. The paper is supported by National Natural Science Foundation of China (No.U1404101).

\section{References}

Hickey, J. B. (1983). Semigroup under a sandwich operation. P Edinburgh Math Soc, 371-382. https://doi.org/10.1017/S0013091500004442

Honyam, P., \& Sanwong J. (2011). Semigroups of transformations with invariant set. J Korean Math Soc, 48, $289-300$. https://doi.org/10.4134/JKMS.2011.48.2.289

Kemprasit, Y., \& Jaidee, S. (2005). Regularity and isomorphism theorems of generalized order-preserving transformation semigroups. $V J M, 253-260$.

Magill, K. D. Jr., \& Subbiah, S. (1975). Green's relations for regular elements of sandwich semigroup (I) general results. $P$ Lond Math Soc, 194-210.

Nenthein, S., Youngkhong, P., \& Kemprasit, Y. (2005). Regular elements of some transformation semigroups. PU M A, 307-314.

Pei, H. S., Sun, L., \& Zhai, H. C. (2007). Green's relations for the variants of transformation semigroups preserving an equivalence relation. Commun Algebra, 1971-1986. https://doi.org/10.1080/00927870701247112

Symons, J. S. (1975). On a generalization of the transformation semigroup. J Aust Math Soc, 47-61. https://doi.org/10.1017/S1446788700023533

Tsyaputa, G. Y. (2004). Green's relations on the deformed transformation semigroups. Algebra D Math, $121-131$.

\section{Copyrights}

Copyright for this article is retained by the author(s), with first publication rights granted to the journal.

This is an open-access article distributed under the terms and conditions of the Creative Commons Attribution license (http://creativecommons.org/licenses/by/4.0/). 